\title{
Wind Direction Manifestation on HF Ocean Radar Echoes
}

\author{
M.L. Heron and P.Marrone ${ }^{1}$ \\ Marine Geophysical Laboratory, James Cook University, \\ Townsville, Queensland, Australia \\ ${ }^{1}$ Daronmont Technologies, \\ Salisbury South, South Australia
}

\begin{abstract}
A direct measure from the spectra of HF radar sea echoes is the ratio of energy in the dominant Bragg peaks. If a single lobe is assumed for the directionality then two radar stations can be used to provide a closed solution for the direction of the lobe. In this work we examine the potential of using just one radar station to derive directional information about wind fields. It is shown that HF radar Bragg ratios are effective in providing timing and location information for features appearing on Mean Sea Level synoptic charts.
\end{abstract}

\section{INTRODUCTION}

The sea echo received on an HF ocean radar is primarily scattered by a Bragg resonance between the radio wave and the sea waves, which produces two high energy spectral lines in the Doppler Shift spectrum of echoes. The first-order Bragg lines in the spectrum are typically over $10 \mathrm{db}$ above the other echo energy, and are typically $20-40 \mathrm{db}$ above the background noise generated by atmospherics in the HF band, shown in Fig. 1 as red dotted lines labeled $f_{B}$ and $-f_{B}$. The energy around the blue dotted line at frequencies above $f_{B}$ and the continuum around zero frequency are from second-order scattering. An analysis based on the first and second-order parts of the Doppler spectrum from two spaced radar stations has been developed to provide estimates of the directional spectrum of sea surface gravity waves (Wyatt, 1990; 2000); and with some assumptions about wave generation, this may be used to estimate wind direction and speed (Heron and Prytz, 2002). In this study we are focusing on the capability of extracting wind direction information using data from a single radar station. There are two main reasons for doing this. One is to improve the overall reliability of an operational twostation system by bridging periods when one station is off for maintenance. The other reason is to examine the capability of a single radar to provide meteorological information in areas where data are sparse.

The process of Bragg scatter involves two conditions to select the two waves on the sea surface which produce the first order spectral lines. One condition is that the sea surface gravity waves must be propagating in the look direction of the radar (which varies from point to point on the surface of the ocean); and the second condition is that the wavelength of the scattering waves is half the wavelength of the radio wave.
There are only two sea waves at any given point on the sea surface which satisfy these conditions; one propagating towards the radar station, and one propagating away.

Usually, one of the Bragg lines has more energy than the other. In Fig. 1, the energy at $f_{B}$ is greater than the energy at $\mathrm{f}_{\mathrm{B}}$ which means, in this case, that the approaching resonant sea surface gravity wave has a greater magnitude, $\mathrm{E}_{+}$, than that of the receding wave, E. In general the ratio of energies in these two waves give us a cut across the wind wave directional spectrum at the fixed wavelength of the resonant sea surface gravity waves. If the wind is blowing towards the radar station, then $\mathrm{E}_{+} / \mathrm{E}_{\text {- }}$ is very large; if the wind is blowing perpendicular to the look direction of the radar, then $\mathrm{E}_{+} / \mathrm{E}_{-}=1$, and if the wind is blowing away from the radar station then $\mathrm{E}_{+} / \mathrm{E}_{\text {- }}$ is close to zero.

If we assume a form for the directional wave spectrum at this wavelength, like that given by equation (1) and shown in Fig. 2, then we can determine the direction of the main lobe. In this model, we assume that the direction of the main lobe of the wave spectrum is also the direction of the wind. If $\mathrm{E}_{+} / \mathrm{E}_{-}$is observed, then the direction of the wind can be estimated with an unresolved ambiguity indicated in Fig. 2. Heron and Rose (1986) used single stations and different azimuths to remove the ambiguity under the assumption that the wind field would vary only in the offshore direction perpendicular to the straight coast.

$$
G(k, \theta)=A(k) \cos ^{2 S}\left(0.5\left(\theta-\theta_{0}\right)\right)
$$

Where $A(k)$ is chosen so that $\int_{0}^{2 \pi} G(k, \pi) \mathrm{d} \theta=1$.

If two HF radar stations are operating over the same area of sea, then the ambiguity shown in Fig. 2 can be resolved and a wind direction can be determined for that time and location, subject only to the limitations of the model. The adoption of one model or another becomes a significant issue in quality control of these data and the ACORN primary data archive avoids this by saving the ratio $\mathrm{E}_{+} / \mathrm{E}_{-}$for each station, time and grid point. 


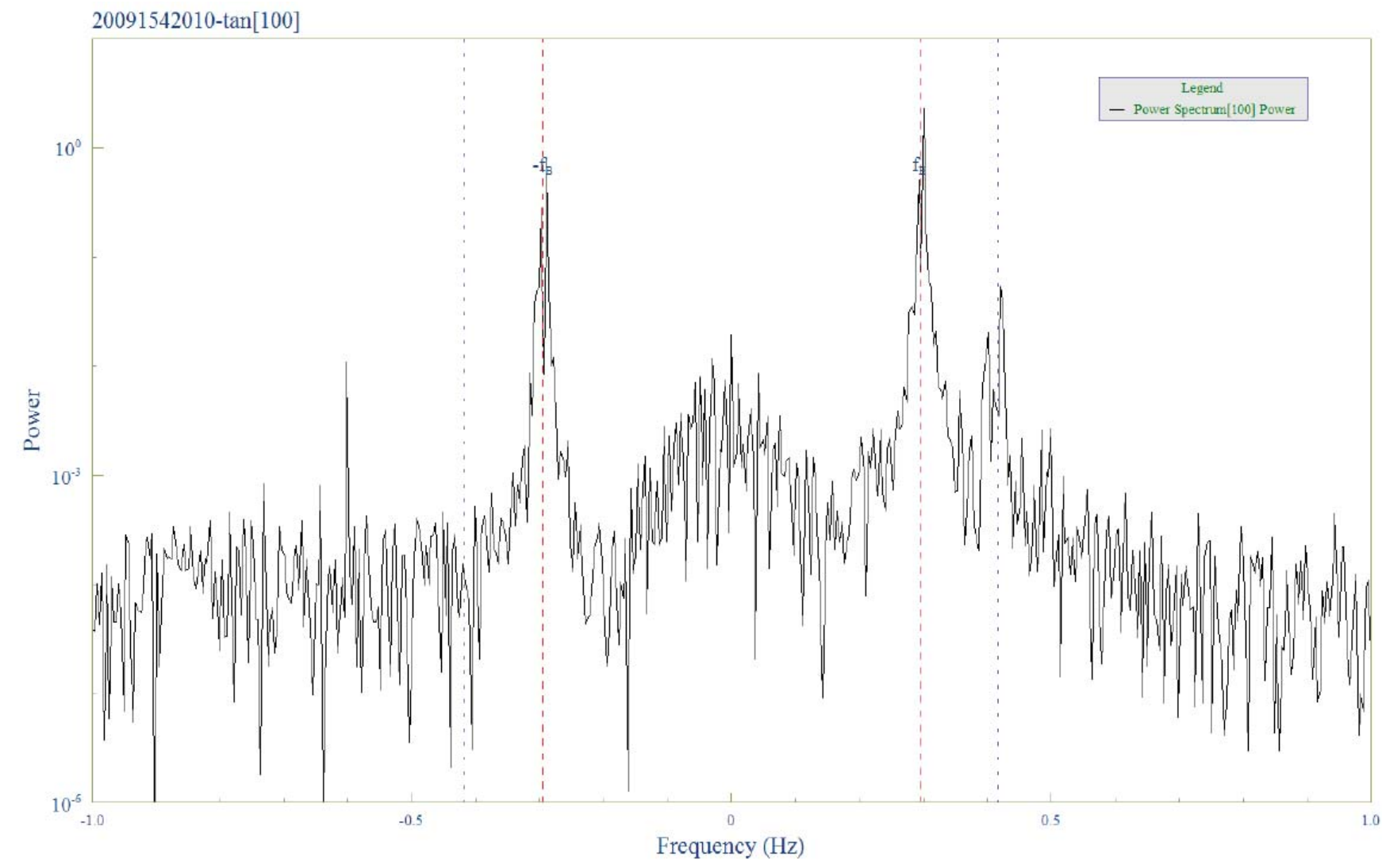

Figure 1. Typical Doppler Shift spectrum for sea echoes on a beam-forming phased array HF ocean surface radar operating at $8.348 \mathrm{MHz}$. The red lines labeled $f_{B}$ and $-f_{B}$ are produced from first-order Bragg scatter.

\section{DATA}

The HF radar data used in this study were taken from the archive of the Integrated Marine Observing System (IMOS) for the Cape Wiles (CWI) station at the southern tip of the Eyre Peninsula in South Australia $\left(34^{\mathrm{O}} 56.60^{\prime} \mathrm{S}, 135^{\mathrm{O}} 41.04^{\prime}\right.$ E). The Cape Wiles radar is a phased array system operating at $8.521 \mathrm{MHz}$ for alternating 5-minute periods. From the netCDF archive, we have extracted the Bragg energies $\left(\mathrm{E}_{+}\right.$and E.) for the two peaks, and significant wave heights on a sparse $80 \times 80$ grid of points on a geographic grid of equal increments in longitude and latitude (at approximately $4 \mathrm{~km}$ spacing) for a 25-hour period centered on 0000 UTC 30 Jan 2009. Data files are stamped with the commencement time of the 5-minute sampling period; for the Cape Wiles radar station, this is on each 10-minute tick in Universal Time.

At each grid point, ratios were calculated as:

$$
\mathrm{R}=10 \log _{10}\left(\mathrm{E}_{+} / \mathrm{E}_{-}\right),
$$

and simple averages were calculated for the 7 records with start times 30 minutes before the hour to 30 minutes after the hour. We stamp that average with the time on the hour. An

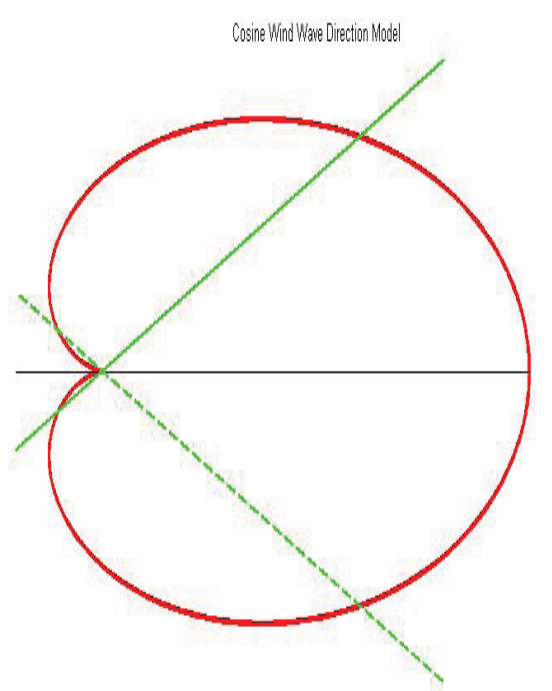

Figure 2. The Cosine wind direction model showing a radar look direction

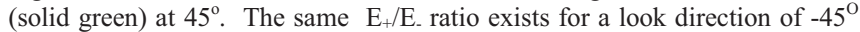
and this creates an ambiguity. 


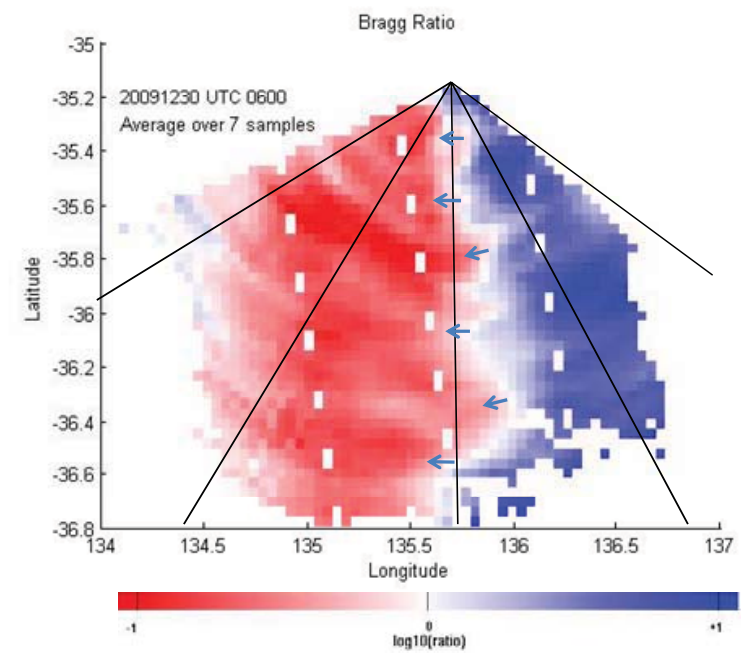

(a)

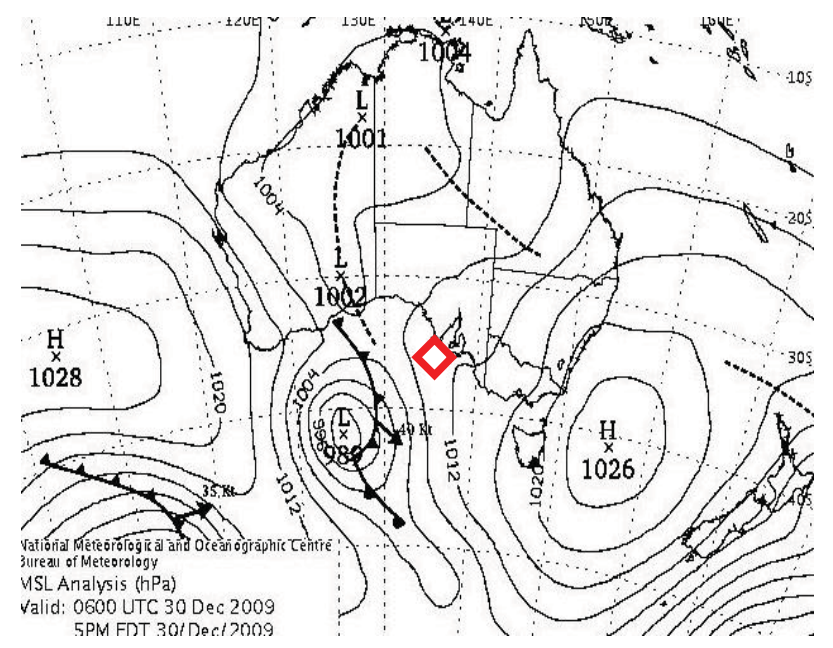

(b)

Figure 3. (a) A 1-hour average of $\log _{10}$ (ratio) with blue showing areas where the dominant Bragg wave has a component towards the Cape Wile radar station (at the vertex), and red where the dominant Bragg wave has a component receding from the station. The black radial lines from the station are drawn to help in this interpretation. On the transition line (white area) the wind is orthogonal to the radial from the station and is indicated with small blue arrows. These data indicate that the wind over the area of observation is fairly uniform and from about $8^{\mathrm{O}}$ north of east. (b) The MSL chart at the corresponding time, with a red square showing the observation area for the Cape Wiles radar.

example of a one-hour average of Bragg ratios is shown in Fig. 3 (a), with a set of direction radials from the Cape Wiles radar station superposed. The blue shaded area indicates that the wind vector has a component in the radial direction towards the radar station, and the red shaded area indicates that there is a component receding from the radar. The radial lines help to visualize the wind field.

The period was chosen to correspond to the passage of an abrupt change in the wind field across the region, accompanied by a strong and clear cold front system showing on the Mean Sea Level (MSL) analysis charts. The MSL charts were obtained from the archive of the Australian Bureau of Meteorology and are available at 6-hourly intervals. Fig. 3(b) shows the MSL chart for 0600UTC on 30 Dec 2009, with the area covered by the radar marked with a red square.

\section{RESULTS}

\section{A. Comparing the radar data and MSL chart}

In Fig. 3(a), the Bragg ratio, $\mathrm{R}$, calculated as in equation (2) is colour coded. It is difficult to derive information from the deeply coloured zones, but the overall colour pattern in Fig. 3(a) is generally consistent with a uniform wind field from about $8^{\mathrm{O}}$ north of east. The white transition zone between red and blue holds special significance and lends itself to a more precise interpretation. At all points along the white transition zone, the wind direction is perpendicular to the local radial to the radar station. The small blue arrows have been drawn on the diagram to indicate the wind directions in the transition zone. Clearly the overall wind field across the area is directed from a few degrees north of east.

The MSL chart shown in Fig. 3(b) is generally consistent with the colour map of Fig. 3(a) where an easterly will have a component towards the radar station on the eastern side (blue) and a component away from the station on the western side of the area. It is easier to see this when some radials are shown, and these are the black lines on the diagram.

\section{B. Six-hourly samples through a front}

Fig. 4 shows the progression in 6-hour steps towards the arrival of the frontal change some time before midnight. At $1200 \mathrm{~h}$ (top panel), the wind across the area remains at eastnorth-east according to the radar, while the MSL chart suggests a more northerly wind.

At 1800 UTC on 30 Dec 2009 (Fig.4 middle panel), the radar estimate of wind direction remains dominantly from the east, while the MSL chart indicates that the low pressure saddle is over the observation area.

At 2400 UTC 30 Dec 2009 (Fig. 4 bottom panel) the radar pattern indicates that the wind is from the south-easterly quarter. The MSL chart indicates that the low pressure saddle has already moved to the east of the observation area, and there is cold front activity over the observation area. 


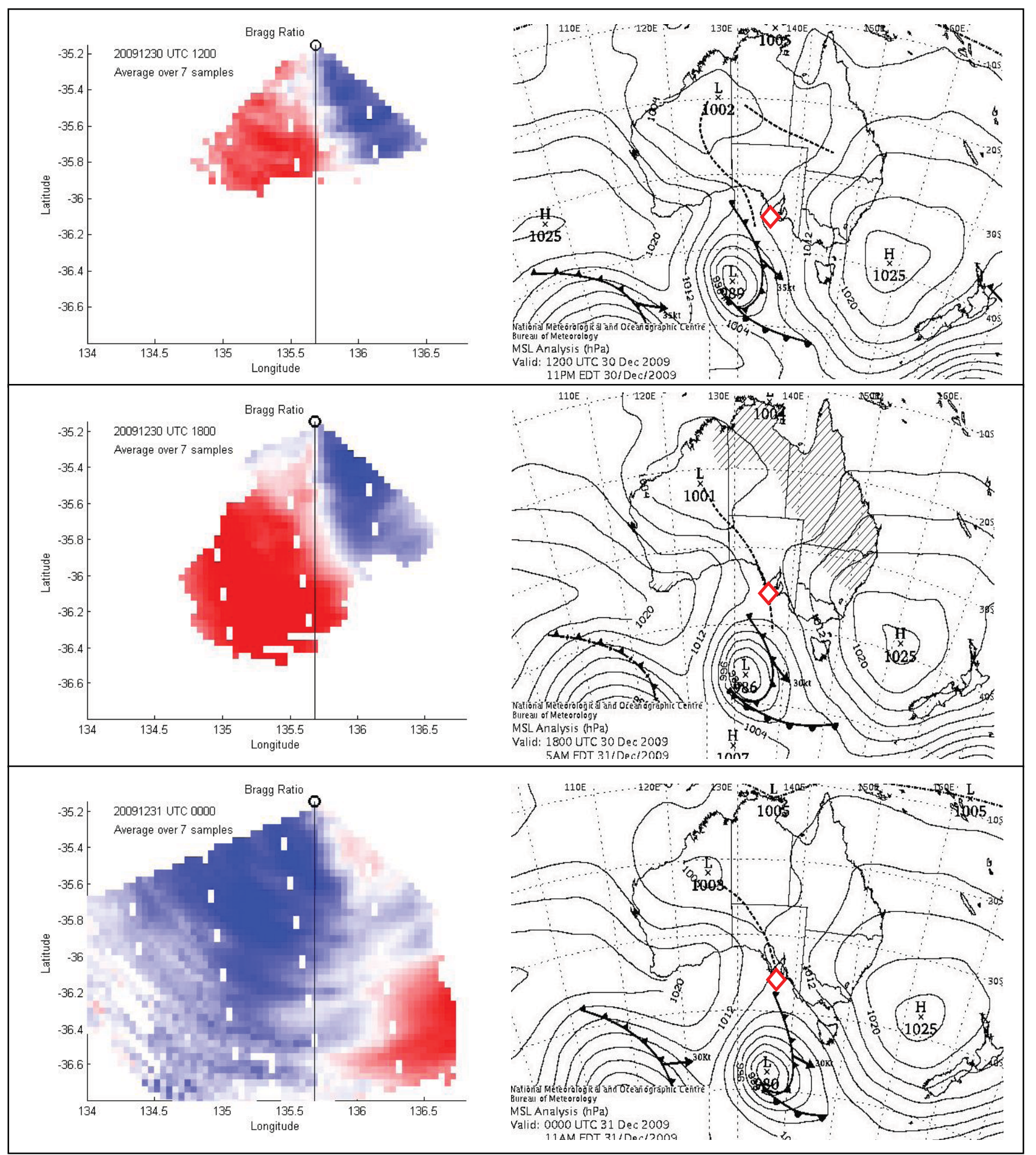

Figure 4. Radar directional ratios plotted as $\log _{10}$ (ratio) on the left with the MSL chart on the right, for 1200, 1800 and 2400 on 30 Dec 2009 (UTC). The front passed the longitude of the radar station just before midnight on 30 Dec 2009 (UTC). 


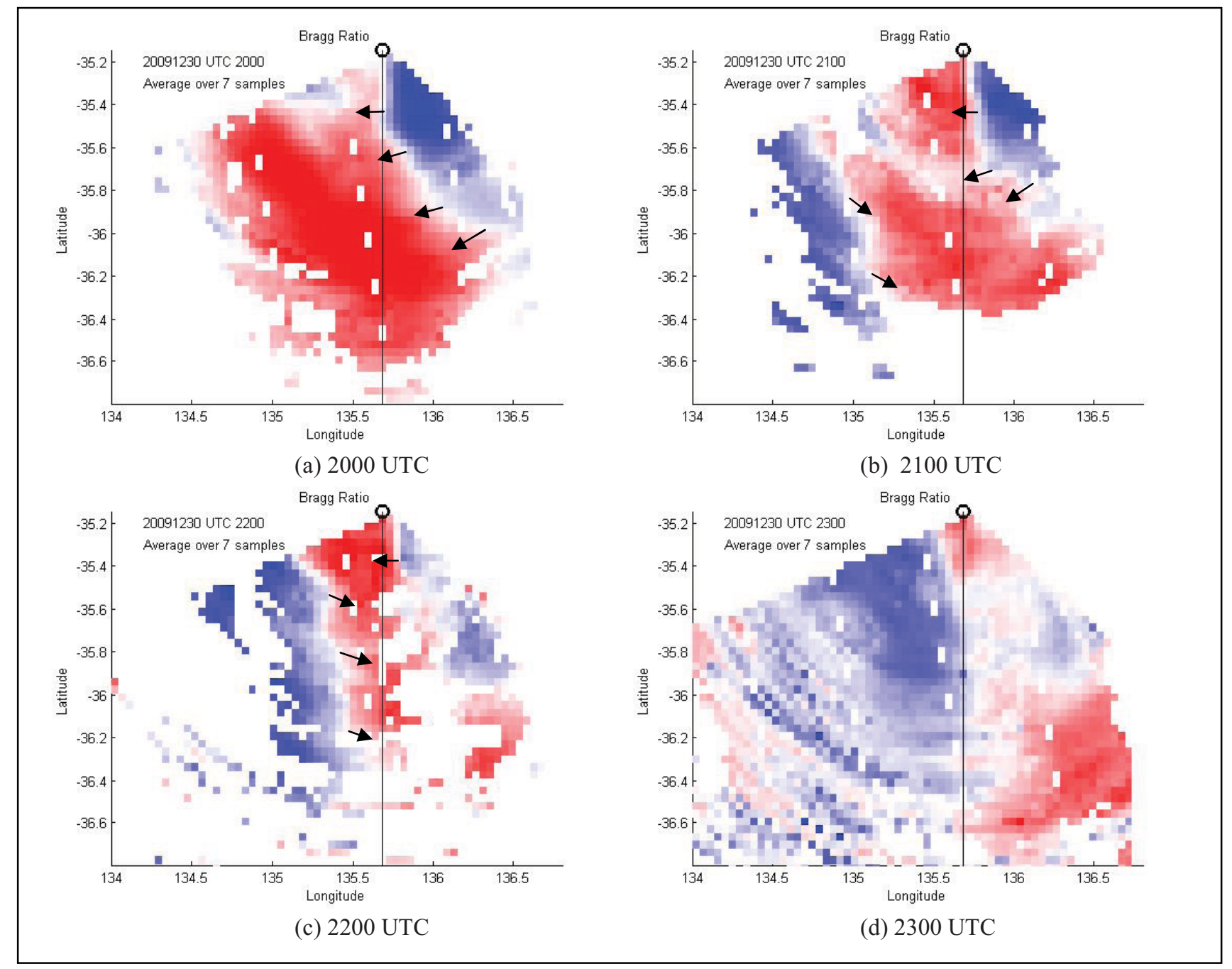

Figure 5. Hourly steps of HF radar Bragg ratios during the passage of a front. (a) 2000 UTC 30 Dec 2009; (b) 2100 UTC 30 Dec 2009 ; (c) 2200 UTC 30 Dec 2009; (d) 2300 UTC 30 Dec 2009.

Next we will look at hourly steps through the change.

\section{Hourly samples of radar data through the front}

At 2000 UTC (Fig. 5(a)), the dominant wind direction is from the north-east over most of the area, with an easterly indicated within about $30 \mathrm{~km}$ of the radar station (and the coast).

At 2100 UTC (Fig. 5(b)), the wind has a northerly component (away from the radar) at all points on the transect line of constant longitude through the radar station. Note that on the eastern side at short range, and on the western side at long ranges, there are wind components directed radially towards the radar. At the white transition zones, the wind is perpendicular to the radial from the station to the point; where the transition zone is clear, we have drawn the wind directions and they indicate a north-easterly to the east of Cape Wiles, and a north-westerly on the west side.

At 2200 UTC (Fig. 5(c)), the wind on the longitude transect has a consistent southerly component, and the clear transition zone to the west of Cape Wiles indicates a north-westerly wind.At 2300 UTC (Fig. 5(d)), the wind along the longitude transect has a northerly component at all but the shortest ranges. At this time, the transition zones are indistinct. The blue shaded area on the western side of the map indicates that the air mass on the western side has a component of drift towards the radar station site. The balance of red shading on the eastern side indicates a general eastwards flow of air across the region. At this time the front has passed the longitude of Cape Wiles. 


\section{CONCLUSION}

The case study presented here shows that the basic observed parameter, $\mathrm{E}_{+} / \mathrm{E}_{-}$, is a useful indicator of wind patterns over the ocean. The key to success here is to acknowledge that data points for wind directions can be determined (with a 180 degree ambiguity) whenever the ratio is unity (in the analysis here we used $\log _{10}$ (ratio) to get symmetry about zero). In the case study, the passage of a frontal system seen on the Mean Sea Level meteorological charts coincided with a wind direction change of nearly 180 degrees.

In situations where data from only one radar station are available, the use of the ratio $\mathrm{E}_{+} / \mathrm{E}_{-}$allows wind direction information to be extracted in a useful way for real time operations. The use of Bragg Ratios from one radar station is a quick way to identify abrupt changes in wind direction as mesoscale weather patterns cross the area. The operation of only one station might happen if one of a pair is unserviceable for a period.

If a need arose for wind direction observations in a remote maritime region, then it might be economical to install only one radar. This would also have the advantage of providing other single-station radar data like radial current components and wave heights. One such location is the Great Australian Bight to the west of Cape Wiles and the Eyre Peninsula in Australia. This work has been undertaken to evaluate the potential of the ex Defence SECAR radar to map the location and movement of meteorological fronts over the eastern half of the Great Australian Bight. The SECAR radar is capable of producing data of this type to ranges of 500-600 km.

\section{ACKNOWLEDGMENT}

This work forms part of the Integrated Marine Observing System within the National Collaborative Research Infrastructure Strategy in Australia, and all radar data are from the IMOS archive. Meteorological MSL charts were supplied by the Bureau of Meteorology, Australia.

\section{REFERENCES}

Heron, M.L. and R.J. Rose, On the application of HF radar to the observation of temporal and spatial changes in wind direction, IEEE Journal of Oceanic Engineering, OE-11, 210218, 1986.

Heron, M.L. and A. Prytz, Wave height and wind direction from HF coastal ocean surface radar, Canadian Journal of Remote Sensing, 28, 385-393, 2002.

Wyatt, L., A relaxation method for integral inversion applied to HF radar measurement of the ocean wave directional spectrum, International Journal of Remote Sensing, 11, 14811494, 1990.

Wyatt, L.R., Limits to the inversion of HF radar backscatter for ocean wave measurement, Journal of Atmospheric and Oceanic Technology, 17, 1651-1666, 2000. 